\title{
Gustafson-Rakha-Type Elliptic Hypergeometric Series
}

\author{
Hjalmar ROSENGREN
}

Department of Mathematical Sciences, Chalmers University of Technology and

University of Gothenburg, SE-412 96 Gothenburg, Sweden

E-mail: hjalmar@chalmers.se

URL: http://www. math.chalmers.se/ hjalmar/

Received February 02, 2017, in final form May 29, 2017; Published online June 01, 2017

https://doi.org/10.3842/SIGMA.2017.037

\begin{abstract}
We prove a multivariable elliptic extension of Jackson's summation formula conjectured by Spiridonov. The trigonometric limit case of this result is due to Gustafson and Rakha. As applications, we obtain two further multivariable elliptic Jackson summations and two multivariable elliptic Bailey transformations. The latter four results are all new even in the trigonometric case.
\end{abstract}

Key words: elliptic hypergeometric series; multivariable hypergeometric series; Jackson summation; Bailey transformation

2010 Mathematics Subject Classification: 33D67

\section{Introduction}

By combining two integral evaluations previously obtained by Gustafson [6], Gustafson and Rakha [7] evaluated the basic hypergeometric integral

$$
\int \frac{\prod_{1 \leq i<j \leq n}\left(z_{i} / z_{j}\right)_{\infty}\left(z_{j} / z_{i}\right)_{\infty} \prod_{j=1}^{n}\left(S / z_{j}\right)_{\infty}}{\prod_{1 \leq i<j \leq n}\left(t z_{i} z_{j}\right)_{\infty} \prod_{i=1}^{n}\left(\prod_{j=1}^{3}\left(c_{j} z_{i}\right)_{\infty} \prod_{j=1}^{n}\left(d_{j} / z_{i}\right)_{\infty}\right)} \frac{d z_{1}}{z_{1}} \cdots \frac{d z_{n-1}}{z_{n-1}}
$$

where the integration is over $\left|z_{1}\right|=\cdots=\left|z_{n-1}\right|=1,(z)_{\infty}=\prod_{j=0}^{\infty}\left(1-q^{j} z\right)$, the parameters satisfy

$$
|q|,|t|,\left|c_{1}\right|,\left|c_{2}\right|,\left|c_{3}\right|,\left|d_{1}\right|, \ldots,\left|d_{n}\right|<1
$$

$z_{n}$ is determined from the integration variables through $z_{1} \cdots z_{n}=1$ and $S=t^{n-2} c_{1} c_{2} c_{3} d_{1} \cdots d_{n}$. By applying residue calculus to (1.1), they could evaluate a certain multivariable basic hypergeometric finite sum, equivalent to the case $p=0$ of Theorem 3.1 below.

Since the seminal work of Date et al. [4] and Frenkel and Turaev [5], it has been recognized that basic hypergeometric functions appear as the trigonometric limit of more general elliptic hypergeometric functions. Elliptic extensions of Gustafson's two integral evaluations mentioned above were conjectured in $[16,19]$ and proved in [10]. Spiridonov [16] used these (at the time conjectural) evaluations to obtain an elliptic extension of (1.1). He also stated the corresponding summation formula as a conjecture. Although it seems likely that this conjecture can be deduced from Spiridonov's integral, such a derivation is still missing from the literature. The purpose of

This paper is a contribution to the Special Issue on Elliptic Hypergeometric Functions and Their Applications. The full collection is available at https://www.emis.de/journals/SIGMA/EHF2017.html 
the present paper is to give a direct proof of Spiridonov's conjectured summation and to apply it to derive some further summation and transformation formulas.

It is worth mentioning that Spiridonov's elliptic extension of (1.1) can be interpreted as the identity between superconformal indices of two dual quantum field theories [17, Sections 12.1.212.1.3]. This indicates that (1.1) and related results are not mere curiosities and that it is not unreasonable to expect further applications.

The plan of the paper is as follows. In Section 3, we prove Spiridonov's conjecture. The proof is elementary and provides in particular a significant simplification of the trigonometric case. The only previously known proof of the Gustafson-Rakha summation is the original one, which as we recall is based on first proving two auxiliary multiple integral evaluations, combining them to obtain (1.1) and finally on a technical computation to pass from integrals to finite residue sums. In Section 4, we give some applications of our result. Namely, combining the elliptic Gustafson-Rakha sum with a summation from [14], we obtain two transformation formulas and two further summation formulas for multivariable elliptic hypergeometric series. These four results are all new even in the trigonometric case.

Note added in proof: After completing this work, I learned from Masahiko Ito and Masatoshi Noumi that they have independently proved Theorem 3.1, using a different method.

\section{Preliminaries}

When $z=\left(z_{1}, \ldots, z_{n}\right)$ is a vector we will write $|z|=z_{1}+\cdots+z_{n}$ and $Z=z_{1} \cdots z_{n}$.

Throughout, $p$ and $q$ will be fixed parameters with $|p|<1$. We employ the standard notation

$$
\begin{aligned}
\theta(z) & =\prod_{j=0}^{\infty}\left(1-p^{j} z\right)\left(1-p^{j+1} / z\right), \\
(z)_{k} & = \begin{cases}\theta(z) \theta(q z) \cdots \theta\left(q^{k-1} z\right), & k \in \mathbb{Z}_{\geq 0}, \\
1 / \theta\left(q^{k} z\right) \theta\left(q^{k+1} z\right) \cdots \theta\left(q^{-1} z\right), & k \in \mathbb{Z}_{<0}\end{cases}
\end{aligned}
$$

as well as

$$
\theta\left(z_{1}, \ldots, z_{m}\right)=\theta\left(z_{1}\right) \cdots \theta\left(z_{m}\right), \quad\left(z_{1}, \ldots, z_{m}\right)_{k}=\left(z_{1}\right)_{k} \cdots\left(z_{m}\right)_{k}
$$

Most of our computations are based on the elementary identities

$$
\begin{aligned}
& \theta(1 / z)=\theta(p z)=-\theta(z) / z \\
& (a)_{n+k}=(a)_{n}\left(a q^{n}\right)_{k}, \quad(a)_{n-k}=(-1)^{k} q^{\left(\begin{array}{c}
k \\
2
\end{array}\right)\left(q^{1-n} / a\right)^{k}} \frac{(a)_{n}}{\left(q^{1-n} / a\right)_{k}},
\end{aligned}
$$

which will be used without comment. All our sums contain the $A$-type factor

$$
\frac{\Delta\left(z q^{x}\right)}{\Delta(z)}=\prod_{1 \leq i<j \leq n} \frac{q^{x_{i}} \theta\left(q^{x_{j}-x_{i}} z_{j} / z_{i}\right)}{\theta\left(z_{j} / z_{i}\right)}
$$

where $z=\left(z_{1}, \ldots, z_{n}\right)$ and $x=\left(x_{1}, \ldots, x_{n}\right)$ is the summation index. We mention the useful identity [12, equation (3.8)]

$$
\frac{\Delta\left(z q^{x}\right)}{\Delta(z)}=(-1)^{|x|} q^{-\left(\begin{array}{c}
|x| \\
2
\end{array}\right)-|x|} \prod_{i, j=1}^{n} \frac{\left(q z_{i} / z_{j}\right)_{x_{i}}}{\left(q^{-x_{j}} z_{i} / z_{j}\right)_{x_{i}}}
$$


and [21, Example 20.53.3]

$$
\sum_{k=1}^{n} \frac{\prod_{j=1}^{n+1} \theta\left(z_{k} / b_{j}\right)}{\theta\left(z_{k} / t\right) \prod_{j=1, j \neq k}^{n} \theta\left(z_{k} / z_{j}\right)}=\frac{\prod_{j=1}^{n+1} \theta\left(b_{j} / t\right)}{\prod_{j=1}^{n} \theta\left(z_{j} / t\right)}
$$

valid for $t z_{1} \cdots z_{n}=b_{1} \cdots b_{n+1}$.

In the one-variable case, the most fundamental results for elliptic hypergeometric series are the elliptic Jackson (or Frenkel-Turaev) summation

$$
\sum_{x=0}^{N} \frac{\theta\left(a q^{2 x}\right)}{\theta(a)} \frac{\left(a, b, c, d, e, q^{-N}\right)_{x} q^{x}}{\left(q, a q / b, a q / c, a q / d, a q / e, a q^{N+1}\right)_{x}}=\frac{(a q, a q / b c, a q / b d, a q / c d)_{N}}{(a q / b, a q / c, a q / d, a q / b c d)_{N}},
$$

valid for $a^{2} q^{N+1}=b c d e$, and the elliptic Bailey transformation

$$
\begin{aligned}
& \sum_{x=0}^{N} \frac{\theta\left(a q^{2 x}\right)}{\theta(a)} \frac{\left(a, b, c, d, e, f, g, q^{-N}\right)_{x} q^{x}}{\left(q, a q / b, a q / c, a q / d, a q / e, a q / f, a q / g, a q^{N+1}\right)_{x}} \\
& =\frac{(a q, a q / e f, \lambda q / e, \lambda q / f)_{N}}{(\lambda q, \lambda q / e f, a q / e, a q / f)_{N}} \sum_{x=0}^{N} \frac{\theta\left(\lambda q^{2 x}\right)}{\theta(\lambda)} \frac{\left(\lambda, \lambda b / a, \lambda c / a, \lambda d / a, e, f, g, q^{-N}\right)_{x} q^{x}}{\left(q, a q / b, a q / c, a q / d, \lambda q / e, \lambda q / f, \lambda q / g, \lambda q^{N+1}\right)_{x}}
\end{aligned}
$$

valid for $a^{3} q^{N+2}=b c d e f g, \lambda=a^{2} q / b c d[5]$. Numerous multivariable extensions of (2.3) and (2.4) are known, see, e.g., $[3,9,11,12,13,14,18,20]$; further examples are obtained in the present paper. We will need one such result, a multivariable extension of (2.3) obtained in [14] (see [15] for the case $p=0)$. Namely, for $a^{2} q^{|N|+1}=b c d e$,

$$
\begin{gathered}
\sum_{x_{1}, \ldots, x_{n}=0}^{N_{1}, \ldots, N_{n}} \frac{\Delta\left(z q^{x}\right)}{\Delta(z)} \frac{\theta\left(a q^{2|x|}\right)}{\theta(a)} \frac{(a, b, c)_{|x|} \prod_{i=1}^{n}\left(d / z_{i}\right)_{|x|}}{\left(a q / b, a q / c, a q^{|N|+1}\right)_{|x|} \prod_{i=1}^{n}\left(a q^{\left.|N|+1-N_{i} / e z_{i}\right)_{|x|}}\right.} q^{|x|} \\
\quad \times \prod_{i=1}^{n} \frac{\left(a q^{|N|+1} / e z_{i}\right)_{|x|-x_{i}}\left(e z_{i}\right)_{x_{i}} \prod_{j=1}^{n}\left(q^{-N_{j}} z_{i} / z_{j}\right)_{x_{i}}}{\left(d / z_{i}\right)_{|x|-x_{i}}\left(a q z_{i} / d\right)_{x_{i}} \prod_{j=1}^{n}\left(q z_{i} / z_{j}\right)_{x_{i}}} \\
=\frac{(a q, a q / b c)_{|N|}}{(a q / b, a q / c)_{|N|}} \prod_{i=1}^{n} \frac{\left(a q z_{i} / b d, a q z_{i} / c d\right)_{N_{i}}}{\left(a q z_{i} / d, a q z_{i} / b c d\right)_{N_{i}}}
\end{gathered}
$$

\section{The elliptic Gustafson-Rakha summation}

Our main result is the following identity. It is easy to see that the case $p=0$ is equivalent to $[7$, Theorem 1.2] and the general case to the conjecture of $[16$, p. 953]. Recall the notation $Z=z_{1} \cdots z_{n}$.

Theorem 3.1. For parameters subject to $q^{N-1} b_{1} \cdots b_{4} z_{1}^{2} \cdots z_{n}^{2}=1$,

$$
\sum_{\substack{x_{1}, \ldots, x_{n} \geq 0, x_{1}+\cdots+x_{n}=N}} \frac{\Delta\left(z q^{x}\right)}{\Delta(z)} \prod_{1 \leq i<j \leq n} q^{x_{i} x_{j}}\left(z_{i} z_{j}\right)_{x_{i}+x_{j}} \prod_{i=1}^{n} \frac{\prod_{j=1}^{4}\left(z_{i} b_{j}\right)_{x_{i}}}{z_{i}^{x_{i}} \prod_{j=1}^{n}\left(q z_{i} / z_{j}\right)_{x_{i}}}
$$




$$
= \begin{cases}\frac{\left(Z b_{1}, Z b_{2}, Z b_{3}, Z b_{4}\right)_{N}}{Z^{N}(q)_{N}}, & n \text { odd }, \\ \frac{\left(Z, Z b_{1} b_{2}, Z b_{1} b_{3}, Z b_{1} b_{4}\right)_{N}}{\left(Z b_{1}\right)^{N}(q)_{N}}, & n \text { even } .\end{cases}
$$

We will prove Theorem 3.1 by induction on $N$. In the case $N=1$, we have $x_{i}=\delta_{i k}$ for some $k$. Using $k$ as summation index, Theorem 3.1 reduces to the following theta function identity.

Lemma 3.2. For parameters subject to $b_{1} b_{2} b_{3} b_{4} z_{1}^{2} \cdots z_{n}^{2}=1$,

$$
\sum_{k=1}^{n} \frac{\prod_{j=1}^{4} \theta\left(z_{k} b_{j}\right)}{z_{k}} \prod_{j=1, j \neq k}^{n} \frac{\theta\left(z_{k} z_{j}\right)}{\theta\left(z_{k} / z_{j}\right)}= \begin{cases}\theta\left(Z b_{1}, Z b_{2}, Z b_{3}, Z b_{4}\right) / Z, & n \text { odd }, \\ \theta\left(Z, Z b_{1} b_{2}, Z b_{1} b_{3}, Z b_{1} b_{4}\right) / Z b_{1}, & n \text { even } .\end{cases}
$$

Proof. We apply induction on $n$, starting from the trivial case $n=1$. Let $b_{1}=v w$ and $b_{2}=v / w$, with $v$ and $w$ free parameters. As a function of $w$, each term in the sum, as well as the right-hand side, has the form $f(w)=C \theta(a w, a / w)$ with $C$ and $a$ independent of $w$. It is a classical fact that any such function is determined by its values at two generic points. Indeed, Weierstrass' identity (which is equivalent to the case $n=2$ of (2.2)) states that

$$
f(w)=f(b) \frac{\theta(c w, c / w)}{\theta(c b, c / b)}+f(c) \frac{\theta(b w, b / w)}{\theta(b c, b / c)},
$$

provided that $b c, b / c \notin p^{\mathbb{Z}}$. Thus, it suffices to verify (3.2) for two independent values of $b_{1}$. Assuming $n \geq 2$, we choose $b_{1}=1 / z_{n-1}$ and $b_{1}=1 / z_{n}$. By symmetry, it is enough to consider the second case. Then, the term corresponding to $k=n$ cancels and we are reduced to an identity equivalent to (3.2), with $n$ replaced by $n-1$ and $b_{1}$ by $z_{n}$.

We mention that it is not hard to deduce (3.2) from classical theta function identities. Indeed, let $t=b_{n+1}$ in (2.2) (so that the right-hand side vanishes) and then make the substitutions $n \mapsto n+4,\left(z_{1}, \ldots, z_{n}\right) \mapsto\left(z_{1}, \ldots, z_{n}, 1,-1, \sqrt{p},-1 / \sqrt{p}\right),\left(b_{1}, \ldots, b_{n}\right) \mapsto\left(z_{1}^{-1}, \ldots, z_{n}^{-1}, b_{1}^{-1}, b_{2}^{-1}\right.$, $\left.b_{3}^{-1}, b_{4}^{-1}\right)$. Using the elementary identities $\theta\left(z^{2}\right)=\theta(z,-z, \sqrt{p} z,-\sqrt{p} z)$ and $2=\theta(-1, \sqrt{p},-\sqrt{p})$, one may deduce that the left-hand side of (3.2) is equal to

$$
\frac{1}{2}\left((-1)^{n+1} Z \prod_{j=1}^{4} \theta\left(b_{j}\right)+Z \prod_{j=1}^{4} \theta\left(-b_{j}\right)+\frac{1}{\sqrt{p}} \prod_{j=1}^{4} \theta\left(\sqrt{p} b_{j}\right)-\frac{1}{\sqrt{p}} \prod_{j=1}^{4} \theta\left(-\sqrt{p} b_{j}\right)\right) .
$$

The fact that this equals the right-hand side of (3.2) follows from Jacobi's fundamental formulae [21, Section 21.22].

The inductive step in the proof of Theorem 3.1 is almost identical to that of [12, Theorem 5.1]. Denoting the right-hand side of $(3.1)$ by $R_{N}\left(Z ; b_{1}, b_{2}, b_{3}, b_{4}\right)$ (where we for a moment consider $Z$ as a free variable) we observe that, regardless of the parity of $n$,

$$
R_{N+1}\left(Z ; b_{1}, b_{2}, b_{3}, b_{4}\right)=\frac{q^{N} \theta(q)}{\theta\left(q^{N+1}\right)} R_{1}\left(q^{N} Z ; q^{-N} b_{1}, b_{2}, b_{3}, b_{4}\right) R_{N}\left(Z ; q b_{1}, b_{2}, b_{3}, b_{4}\right) .
$$

Assuming (3.1) for fixed $N$, it follows that

$$
R_{N+1}\left(Z ; b_{1}, b_{2}, b_{3}, b_{4}\right)=\frac{q^{N} \theta(q)}{\theta\left(q^{N+1}\right)} R_{1}\left(q^{N} Z ; q^{-N} b_{1}, b_{2}, b_{3}, b_{4}\right)
$$




$$
\times \sum_{\substack{x_{1}, \ldots, x_{n} \geq 0, x_{1}+\cdots+x_{n}=N}} \frac{\Delta\left(z q^{x}\right)}{\Delta(z)} \prod_{1 \leq i<j \leq n} q^{x_{i} x_{j}}\left(z_{i} z_{j}\right)_{x_{i}+x_{j}} \prod_{i=1}^{n} \frac{\left(q z_{i} b_{1}\right)_{x_{i}} \prod_{j=2}^{4}\left(z_{i} b_{j}\right)_{x_{i}}}{z_{i}^{x_{i}} \prod_{j=1}^{n}\left(q z_{i} / z_{j}\right)_{x_{i}}}
$$

where $Z=z_{1} \cdots z_{n}$ and $q^{N} B Z^{2}=1$. We pull the factor $R_{1}$ inside the sum and expand it using (3.1), with $z_{i}$ replaced by $q^{x_{i}} z_{i}$. This gives

$$
\begin{aligned}
R_{N+1}\left(Z ; b_{1}, b_{2}, b_{3}, b_{4}\right) & \frac{q^{N} \theta(q)}{\theta\left(q^{N+1}\right)} \sum_{\substack{x_{1}, \ldots, x_{n} \geq 0, x_{1}+\cdots+x_{n}=N}} \sum_{\substack{y_{1}, \ldots, y_{n} \geq 0, y_{1}+\cdots+y_{n}=1}} \frac{\Delta\left(z q^{x+y}\right)}{\Delta(z)} \prod_{1 \leq i<j \leq n} q^{x_{i} x_{j}}\left(z_{i} z_{j}\right)_{x_{i}+x_{j}+y_{i}+y_{j}} \\
& \times \prod_{i=1}^{n} \frac{\left(q z_{i} b_{1}\right)_{x_{i}}\left(q^{x_{i}-N} z_{i} b_{1}\right)_{y_{i}} \prod_{j=2}^{4}\left(z_{i} b_{j}\right)_{x_{i}+y_{i}}}{z_{i}^{x_{i}}\left(z_{i} q^{x_{i}}\right)^{y_{i}} \prod_{j=1}^{n}\left(q z_{i} / z_{j}\right)_{x_{i}}\left(q^{1+x_{i}-x_{j}} z_{i} / z_{j}\right)_{y_{i}}} \\
= & \frac{q^{N} \theta(q)}{\theta\left(q^{N+1}\right)} \sum_{\substack{x_{1}, \ldots, x_{n} \geq 0, x_{1}+\cdots+x_{n}=N+1}}^{y_{1}, \ldots, y_{n} \geq 0,} \sum_{y_{1}+\cdots+y_{n}=1} \frac{\Delta\left(z q^{x}\right)}{\Delta(z)} \prod_{1 \leq i<j \leq n} q^{x_{i} x_{j}-x_{i} y_{j}-x_{j} y_{i}}\left(z_{i} z_{j}\right)_{x_{i}+x_{j}} \\
& \times \prod_{i=1}^{n} \frac{\left(q z_{i} b_{1}\right)_{x_{i}-y_{i}}\left(q^{x_{i}-y_{i}-N} z_{i} b_{1}\right)_{y_{i}} \prod_{j=2}^{4}\left(z_{i} b_{j}\right)_{x_{i}}}{z_{i}^{x_{i}} q^{\left(x_{i}-y_{i}\right) y_{i}} \prod_{j=1}^{n}\left(q z_{i} / z_{j}\right)_{x_{i}-y_{i}}\left(q^{1+x_{i}-x_{j}-y_{i}+y_{j}} z_{i} / z_{j}\right)_{y_{i}}},
\end{aligned}
$$

where we replaced each $x_{i}$ by $x_{i}-y_{i}$ and used that $y_{i} y_{j}=0$ for $i \neq j$.

By elementary manipulations, using

$$
\prod_{i<j} q^{x_{i} y_{j}+x_{j} y_{i}} \prod_{i} q^{\left(x_{i}-y_{i}\right) y_{i}}=q^{\left(x_{1}+\cdots+x_{n}\right)\left(y_{1}+\cdots+y_{n}\right)-\left(y_{1}^{2}+\cdots+y_{n}^{2}\right)}=q^{(N+1) \cdot 1-1}=q^{N},
$$

the expression above can be rewritten

$$
\begin{aligned}
& R_{N+1}\left(Z ; b_{1}, b_{2}, b_{3}, b_{4}\right) \\
& =\frac{1}{\theta\left(q^{N+1}\right)} \sum_{\substack{x_{1}, \ldots, x_{n} \geq 0, x_{1}+\cdots+x_{n}=N+1}} \frac{\Delta\left(z q^{x}\right)}{\Delta(z)} \prod_{1 \leq i<j \leq n} q^{x_{i} x_{j}}\left(z_{i} z_{j}\right)_{x_{i}+x_{j}} \prod_{i=1}^{n} \frac{\left(q z_{i} b_{1}\right)_{x_{i}} \prod_{j=2}^{4}\left(z_{i} b_{j}\right)_{x_{i}}}{z_{i}^{x_{i}} \prod_{j=1}^{n}\left(q z_{i} / z_{j}\right)_{x_{i}}} \\
& \quad \times \sum_{\substack{y_{1}, \ldots, y_{n} \geq 0, y_{1}+\cdots+y_{n}=1}} \prod_{i=1}^{n} \frac{\left(q^{x_{i}-y_{i}-N} z_{i} b_{1}\right)_{y_{i}} \prod_{j=1}^{n}\left(q^{1+x_{i}-y_{i}} z_{i} / z_{j}\right)_{y_{i}}}{\left(q^{1+x_{i}-y_{i}} z_{i} b_{1}\right)_{y_{i}} \prod_{j=1, j \neq i}^{n}\left(q^{x_{i}-x_{j}} z_{i} / z_{j}\right)_{y_{i}}} .
\end{aligned}
$$

Writing $y_{i}=\delta_{i k}$, the inner sum takes the form

$$
\sum_{k=1}^{n} \frac{\theta\left(q^{x_{k}-N-1} z_{k} b_{1}\right) \prod_{j=1}^{n} \theta\left(q^{x_{k}} z_{k} / z_{j}\right)}{\theta\left(q^{x_{k}} z_{k} b_{1}\right) \prod_{j=1, j \neq k}^{n} \theta\left(q^{x_{k}-x_{j}} z_{k} / z_{j}\right)} .
$$


By (2.2), this can be evaluated as

$$
\theta\left(q^{N+1}\right) \prod_{i=1}^{n} \frac{\theta\left(z_{i} b_{1}\right)}{\theta\left(q^{x_{i}} z_{i} b_{1}\right)}=\theta\left(q^{N+1}\right) \prod_{i=1}^{n} \frac{\left(z_{i} b_{1}\right)_{x_{i}}}{\left(q z_{i} b_{1}\right)_{x_{i}}}
$$

and we arrive at (3.1) with $N$ replaced by $N+1$. This completes the proof of Theorem 3.1.

We will now rewrite (3.1) in a way that hides some of its symmetry but makes it clear that it generalizes the Frenkel-Turaev summation (2.3). To this end, we replace $n$ by $n+1, z_{n+1}$ by $q^{-N} a^{-1}$ and eliminate $x_{n+1}$ from the summation. After routine simplification, we arrive at the following identity.

Corollary 3.3. Assuming $a^{2} q^{N+1}=b_{1} b_{2} b_{3} b_{4} z_{1}^{2} \cdots z_{n}^{2}$,

$$
\begin{aligned}
& \sum_{\substack{x_{1}, \ldots, x_{n} \geq 0, x_{1}+\cdots+x_{n} \leq N}} \frac{\Delta\left(z q^{x}\right)}{\Delta(z)} \prod_{i=1}^{n} \frac{\theta\left(a z_{i} q^{|x|+x_{i}}\right)}{\theta\left(a z_{i}\right)} \frac{\prod_{1 \leq i<j \leq n}\left(z_{i} z_{j}\right)_{x_{i}+x_{j}}}{\prod_{i=1}^{n}\left(a q / z_{i}\right)_{|x|-x_{i}}} \frac{\left(q^{-N}\right)_{|x|} \prod_{i=1}^{n}\left(a z_{i}\right)_{|x|}}{\prod_{j=1}^{4}\left(a q / b_{j}\right)_{|x|}} q^{|x|} \\
& \times \prod_{i=1}^{n} \frac{\prod_{j=1}^{4}\left(z_{i} b_{j}\right)_{x_{i}}}{\left(a q^{N+1} z_{i}\right)_{x_{i}} \prod_{j=1}^{n}\left(q z_{i} / z_{j}\right)_{x_{i}}} \\
& =\frac{\prod_{j=1}^{n}\left(a q z_{j}\right)_{N}}{\left(a q / b_{1}, a q / b_{2}, a q / b_{3}, a q / b_{1} b_{2} b_{3} Z^{2}\right)_{N} \prod_{j=1}^{n}\left(a q / z_{j}\right)_{N}} \\
& \quad \times\left\{\begin{array}{l}
\left(a q / Z, a q / b_{1} b_{2} Z, a q / b_{1} b_{3} Z, a q / b_{2} b_{3} Z\right)_{N}, \quad n \text { odd }, \\
\left(a q / b_{1} Z, a q / b_{2} Z, a q / b_{3} Z, a q / b_{1} b_{2} b_{3} Z\right)_{N}, \quad n \text { even. }
\end{array}\right.
\end{aligned}
$$

\section{Applications}

The elliptic Bailey transformation (2.4) can be derived from the elliptic Jackson summation (2.3). Similar arguments can be used in multivariable situations, see, e.g., [1, 2, 8] for the trigonometric and [12] for the elliptic case. We will use this method to derive a new multivariable elliptic Bailey transformation by combining the two multivariable elliptic Jackson summations (2.5) and (3.3).

Theorem 4.1. Suppose that $a^{3} q^{N+2}=b c d e f g z_{1}^{2} \cdots z_{n}^{2}$ and let $\lambda=a^{2} q / b c d$. Then,

$$
\begin{aligned}
& \sum_{\substack{x_{1}, \ldots, x_{n} \geq 0, x_{1}+\cdots+x_{n} \leq N}} \frac{\Delta\left(z q^{x}\right)}{\Delta(z)} \prod_{i=1}^{n} \frac{\theta\left(a z_{i} q^{|x|+x_{i}}\right)}{\theta\left(a z_{i}\right)} \frac{\prod_{1 \leq i<j \leq n}\left(z_{i} z_{j}\right)_{x_{i}+x_{j}}}{\prod_{i=1}^{n}\left(a q / z_{i}\right)_{|x|-x_{i}}} \\
& \quad \times \frac{\left(q^{-N}, b\right)_{|x|} \prod_{i=1}^{n}\left(a z_{i}\right)_{|x|}}{(a q / c, a q / d, a q / e, a q / f, a q / g)_{|x|}} q^{|x|} \prod_{i=1}^{n} \frac{\left(c z_{i}, d z_{i}, e z_{i}, f z_{i}, g z_{i}\right)_{x_{i}}}{\left(a q^{N+1} z_{i}, a q z_{i} / b\right)_{x_{i}} \prod_{j=1}^{n}\left(q z_{i} / z_{j}\right)_{x_{i}}} \\
& =\frac{z^{N} \prod_{i=1}^{n}\left(a q z_{i}\right)_{N}}{(\lambda q, a q / e, a q / f, a q / g)_{N} \prod_{i=1}^{n}\left(a q / z_{i}\right)_{N}}
\end{aligned}
$$




$$
\begin{aligned}
& \times \sum_{\substack{x_{1}, \ldots, x_{n} \geq 0, x_{1}+\cdots+x_{n} \leq N}} \frac{\Delta\left(z q^{x}\right)}{\Delta(z)} \frac{\theta\left(\lambda q^{2|x|}\right)}{\theta(\lambda)} \frac{\prod_{1 \leq i<j \leq n}\left(z_{i} z_{j}\right)_{x_{i}+x_{j}}}{\prod_{i=1}^{n}\left(\lambda b / a z_{i}\right)_{|x|-x_{i}}} \\
& \times \frac{\left(\lambda, q^{-N}, \lambda c / a, \lambda d / a\right)_{|x|} \prod_{i=1}^{n}\left(\lambda b / a z_{i}\right)_{|x|}}{\left(\lambda q^{N+1}, a q / c, a q / d\right)_{|x|}} q^{|x|} \prod_{i=1}^{n} \frac{\left(e z_{i}, f z_{i}, g z_{i}, q^{-N} z_{i} / a\right)_{x_{i}}}{\left(a q z_{i} / b\right)_{x_{i}} \prod_{j=1}^{n}\left(q z_{i} / z_{j}\right)_{x_{i}}} \\
& \times \begin{cases}\left(\frac{a}{\lambda}\right)^{N} \frac{(a q / Z, \lambda q / e Z, \lambda q / f Z, \lambda q / g Z)_{N}}{\left(q^{-N} Z / a, \lambda q / e Z, \lambda q / f Z, \lambda q / g Z\right)_{|x|}}, & n \text { odd }, \\
\frac{(\lambda q / Z, a q / e Z, a q / f Z, a q / g Z)_{N}}{(\lambda q / Z, \lambda q / e f Z, \lambda q / e g Z, \lambda q / f g Z)_{|x|}}, & n \text { even. }\end{cases}
\end{aligned}
$$

Proof. If we substitute

$$
\left(N_{1}, \ldots, N_{n}, a, b, c, d, e\right) \mapsto\left(x_{1}, \ldots, x_{n}, \lambda, \lambda c / a, \lambda d / a, \lambda b / a, a q^{|x|}\right)
$$

in (2.5), the right-hand side takes the form

$$
\frac{(\lambda q, b)_{|x|}}{(a q / c, a q / d)_{|x|}} \prod_{i=1}^{n} \frac{\left(c z_{i}, d z_{i}\right)_{x_{i}}}{\left(a q z_{i} / b, a z_{i} / \lambda\right)_{x_{i}}} .
$$

Thus, the left-hand side of (4.1) can be expressed as

$$
\begin{aligned}
& \sum_{\substack{x_{1}, \ldots, x_{n} \geq 0, x_{1}+\cdots+x_{n} \leq N}} \frac{\Delta\left(z q^{x}\right)}{\Delta(z)} \prod_{i=1}^{n} \frac{\theta\left(a z_{i} q^{|x|+x_{i}}\right)}{\theta\left(a z_{i}\right)} \frac{\prod_{1 \leq i<j \leq n}\left(z_{i} z_{j}\right)_{x_{i}+x_{j}}}{\prod_{i=1}^{n}\left(a q / z_{i}\right)_{|x|-x_{i}}} \\
& \quad \times \frac{\left(q^{-N}\right)_{|x|} \prod_{i=1}^{n}\left(a z_{i}\right)_{|x|}}{(\lambda q, a q / e, a q / f, a q / g)_{|x|}} q^{|x|} \prod_{i=1}^{n} \frac{\left(a z_{i} / \lambda, e z_{i}, f z_{i}, g z_{i}\right)_{x_{i}}}{\left(a q^{N+1} z_{i}\right)_{x_{i}} \prod_{j=1}^{n}\left(q z_{i} / z_{j}\right)_{x_{i}}} \\
& \quad \times \sum_{y_{1}, \ldots, y_{n}=0}^{x_{1}, \ldots, x_{n}} \frac{\Delta\left(z q^{y}\right)}{\Delta(z)} \frac{\theta\left(\lambda q^{2|y|}\right)}{\theta(\lambda)} \frac{(\lambda, \lambda c / a, \lambda d / a)_{|y|} \prod_{i=1}^{n}\left(\lambda b / a z_{i}\right)_{|y|}}{\left(a q / c, a q / d, \lambda q^{|x|+1}\right)_{|y|} \prod_{i=1}^{n}\left(\lambda q^{\left.1-x_{i} / a z_{i}\right)_{|y|}} q^{|y|}\right.} \\
& \quad \times \prod_{i=1}^{n} \frac{\left(\lambda q / a z_{i}\right)_{|y|-y_{i}}\left(a q^{|x|} z_{i}\right)_{y_{i}} \prod_{j=1}^{n}\left(q^{-x_{j}} z_{i} / z_{j}\right)_{y_{i}}}{\left(\lambda b / a z_{i}\right)_{|y|-y_{i}}\left(a q z_{i} / b\right)_{y_{i}} \prod_{j=1}^{n}\left(q z_{i} / z_{j}\right)_{y_{i}}}
\end{aligned}
$$

We change the order of summation and replace the vector $x$ by $x+y$. Some elementary manipulation, using in particular (2.1), gives

$$
\begin{aligned}
& \sum_{\substack{y_{1}, \ldots, y_{n} \geq 0, y_{1}+\cdots+y_{n} \leq N}} \frac{\Delta\left(z q^{y}\right)}{\Delta(z)} \frac{\theta\left(\lambda q^{2|y|}\right)}{\theta(\lambda)} \frac{\prod_{1 \leq i<j \leq n} q^{-y_{i} y_{j}}\left(z_{i} z_{j}\right)_{y_{i}+y_{j}}}{\prod_{i=1}^{n}\left(a q / z_{i}, \lambda b / a z_{i}\right)_{|y|-y_{i}}} \frac{\prod_{i=1}^{n}\left(a q z_{i}\right)_{|y|+y_{i}}}{(\lambda q)_{2|y|}} \\
& \quad \times \frac{\left(q^{-N}, \lambda, \lambda c / a, \lambda d / a\right)_{|y|} \prod_{i=1}^{n}\left(\lambda b / a z_{i}\right)_{|y|}}{(a q / c, a q / d, a q / e, a q / f, a q / g)_{|y|}}\left(\frac{a q}{\lambda}\right)^{|y|} \prod_{i=1}^{n} \frac{\left(e z_{i}, f z_{i}, g z_{i}\right)_{y_{i}} z_{i}^{y_{i}}}{\left(a q^{N+1} z_{i}, a q z_{i} / b\right)_{y_{i}} \prod_{j=1}^{n}\left(q z_{i} / z_{j}\right)_{y_{i}}}
\end{aligned}
$$




$$
\begin{aligned}
& \times \sum_{\substack{x_{1}, \ldots, x_{n} \geq 0, x_{1}+\cdots+x_{n} \leq N-|y|}} \frac{\Delta\left(z q^{y+x}\right)}{\Delta\left(z q^{y}\right)} \prod_{i=1}^{n} \frac{\theta\left(a z_{i} q^{|y|+y_{i}+|x|+x_{i}}\right)}{\theta\left(a z_{i} q^{|y|+y_{i}}\right)} \frac{\prod_{1 \leq i<j \leq n}\left(z_{i} z_{j} q^{y_{i}+y_{j}}\right)_{x_{i}+x_{j}}}{\prod_{i=1}^{n}\left(a q^{\left.|y|+1-y_{i} / z_{i}\right)_{|x|-x_{i}}}\right.} \\
& \times \frac{\left(q^{|y|-N}\right)_{|x|} \prod_{i=1}^{n}\left(a z_{i} q^{|y|+y_{i}}\right)_{|x|}}{\left(a q^{|y|+1} / e, a q^{|y|+1} / f, a q^{|y|+1} / g, \lambda q^{2|y|+1}\right)} q_{|x|}^{|x|} \\
& \times \prod_{i=1}^{n} \frac{\left(e z_{i} q^{y_{i}}, f z_{i} q^{y_{i}}, g z_{i} q^{y_{i}}, q^{y_{i}-|y|} a z_{i} / \lambda\right)_{x_{i}}}{\left(a q^{N+1+y_{i}} z_{i}\right)_{x_{i}} \prod_{j=1}^{n}\left(q^{1+y_{i}-y_{j}} z_{i} / z_{j}\right)_{x_{i}}}
\end{aligned}
$$

We observe that the inner sum is as in Corollary 3.3, with the substitutions

$$
\left(z_{1}, \ldots, z_{n}, N, a, b_{1}, b_{2}, b_{3}, b_{4}\right) \mapsto\left(z_{1} q^{y_{1}}, \ldots, z_{n} q^{y_{n}}, N-|y|, a q^{|y|}, e, f, g, q^{-|y|} a / \lambda\right) .
$$

When $n$ is odd, the value of this sum can be rewritten

$$
\begin{gathered}
\frac{(a q / Z, a q / e f Z, a q / e g Z, a q / f g Z)_{N-|y|} \prod_{i=1}^{n}\left(a q^{|y|+1+y_{i}} z_{i}\right)_{N-|y|}}{\left(a q^{|y|+1} / e, a q^{|y|+1} / f, a q^{|y|+1} / g, q^{-N-|y|} / \lambda\right)_{N-|y|} \prod_{i=1}^{n}\left(a q^{\left.|y|+1-y_{i} / z_{i}\right)_{N-|y|}}\right.} \\
=z^{N}\left(\frac{a}{\lambda}\right)^{N-|y|} \frac{(a q / Z, \lambda q / e Z, \lambda q / f Z, \lambda q / g Z)_{N} \prod_{i=1}^{n}\left(a q z_{i}\right)_{N}}{(\lambda q, a q / e, a q / f, a q / g)_{N} \prod_{i=1}^{n}\left(a q / z_{i}\right)_{N}} \\
\times \frac{q^{\sum_{i<j} y_{i} y_{j}}(\lambda q)_{2|y|}(a q / e, a q / f, a q / g)_{|y|} \prod_{i=1}^{n}\left(a q / z_{i}\right)_{|y|-y_{i}}\left(a q^{N+1} z_{i}, q^{-N} z_{i} / a\right)_{y_{i}}}{\left(\lambda q^{N+1}, q^{-N} Z / a, \lambda q / e Z, \lambda q / f Z, \lambda q / g Z\right)_{|y|} \prod_{i=1}^{n} z_{i}^{y_{i}}\left(a q z_{i}\right)_{|y|+y_{i}}}
\end{gathered}
$$

which leads to the right-hand side of (4.1). The case of even $n$ is treated similarly.

One may obtain further transformation formulas by iterating Theorem 4.1. We will only give one example, exploiting the fact that the left-hand side of (4.1) is invariant under interchanging $c$ and $e$. In the identity expressing the corresponding symmetry of the right-hand side, we make the substitutions $(\lambda, a, b, c, d) \mapsto\left(a, a^{2} q / b c d, a q / c d, a q / b d, a q / b c\right)$, keeping $e, f, g, z_{1}, \ldots, z_{n}$ fixed. This leads to another multivariable elliptic Bailey transformation.

Corollary 4.2. Suppose that $a^{3} q^{N+2}=b c \operatorname{def} g z_{1}^{2} \cdots z_{n}^{2}$ and let $\lambda=a^{2} q / b d e$. Then,

$$
\begin{aligned}
& \sum_{\substack{x_{1}, \ldots, x_{n} \geq 0, x_{1}+\cdots+x_{n} \leq N}} \frac{\Delta\left(z q^{x}\right)}{\Delta(z)} \frac{\theta\left(a q^{2|x|}\right)}{\theta(a)} \frac{\prod_{1 \leq i<j \leq n}\left(z_{i} z_{j}\right)_{x_{i}+x_{j}}}{\prod_{i=1}^{n}\left(b / z_{i}\right)_{|x|-x_{i}}} \\
& \times \frac{\left(a, q^{-N}, c, d\right)_{|x|} \prod_{i=1}^{n}\left(b / z_{i}\right)_{|x|}}{\left(a q^{N+1}, a q / c, a q / d\right)_{|x|}} q^{|x|} \prod_{i=1}^{n} \frac{\left(e z_{i}, f z_{i}, g z_{i}, a q z_{i} / e f g Z^{2}\right)_{x_{i}}}{\left(a q z_{i} / b\right)_{x_{i}} \prod_{j=1}^{n}\left(q z_{i} / z_{j}\right)_{x_{i}}} \\
& \times\left\{\begin{array}{l}
\frac{1}{(a q / e Z, a q / f Z, a q / g Z, a q / \text { efgZ })_{|x|}}, \quad n \text { odd }, \\
\frac{1}{(a q / Z, a q / \text { ef } Z, a q / e g Z, a q / f g Z)_{|x|}}, \quad n \text { even }
\end{array}\right.
\end{aligned}
$$




$$
\begin{aligned}
= & \frac{(a q, \lambda q / c)_{N}}{(\lambda q, a q / c)_{N}} \sum_{\substack{x_{1}, \ldots, x_{n} \geq 0, x_{1}+\cdots+x_{n} \leq N}} \frac{\Delta\left(z q^{x}\right)}{\Delta(z)} \frac{\theta\left(\lambda q^{2|x|}\right)}{\theta(\lambda)} \frac{\prod_{1 \leq i<j \leq n}\left(z_{i} z_{j}\right)_{x_{i}+x_{j}}}{\prod_{i=1}^{n}\left(\lambda b / a z_{i}\right)_{|x|-x_{i}}} \\
& \times \frac{\left(\lambda, q^{-N}, c, \lambda d / a\right)_{|x|} \prod_{i=1}^{n}\left(\lambda b / a z_{i}\right)_{|x|}}{\left(\lambda q^{N+1}, \lambda q / c, a q / d\right)_{|x|}} q^{|x|} \prod_{i=1}^{n} \frac{\left(\lambda e z_{i} / a, f z_{i}, g z_{i}, a q z_{i} / e f g Z^{2}\right)_{x_{i}}}{\left(a q z_{i} / b\right)_{x_{i}} \prod_{j=1}^{n}\left(q z_{i} / z_{j}\right)_{x_{i}}} \\
& \times\left\{\begin{array}{cc}
\frac{(a q / c f Z, \lambda q / f Z)_{N}}{(a q / f Z, \lambda q / c f Z)_{N}(a q / e Z, \lambda q / f Z, \lambda q / g Z, a q / e f g Z)_{|x|} \mid}, & n \text { odd }, \\
\frac{(a q / c Z, \lambda q / Z)_{N}}{(a q / Z, \lambda q / c Z)_{N}(\lambda q / Z, a q / e f Z, a q / e g Z, \lambda q / f g Z)_{|x|}}, & n \text { even. }
\end{array}\right.
\end{aligned}
$$

Theorem 4.1 reduces to Corollary 3.3 when $a q=b c$. More interestingly, when $b=1$ the left-hand side of (4.1) reduces to 1. After a change of parameters, this leads to the following new multivariable elliptic Jackson summation.

Corollary 4.3. If $a^{2} q^{N+1}=b c d e z_{1}^{2} \cdots z_{n}^{2}$, then

$$
\begin{aligned}
& \sum_{\substack{x_{1}, \ldots, x_{n} \geq 0 \\
x_{1}+\cdots+x_{n} \leq N}} \frac{\Delta\left(z q^{x}\right)}{\Delta(z)} \frac{\theta\left(a q^{2|x|}\right)}{\theta(a)} \frac{\prod_{1 \leq i<j \leq n}\left(z_{i} z_{j}\right)_{x_{i}+x_{j}}}{\prod_{i=1}^{n}\left(e / z_{i}\right)_{|x|-x_{i}}} \frac{\left(a, q^{-N}\right)_{|x|} \prod_{i=1}^{n}\left(e / z_{i}\right)_{|x|}}{\left(a q^{N+1}\right)_{|x|}} q^{|x|} \\
& \times \prod_{i=1}^{n} \frac{\left(b z_{i}, c z_{i}, d z_{i}, q^{-N} e z_{i} / a\right)_{x_{i}}}{\left(a q z_{i} / e\right)_{x_{i}} \prod_{j=1}^{n}\left(q z_{i} / z_{j}\right)_{x_{i}}} \cdot \begin{cases}\frac{1}{\left(q^{-N} e Z / a, a q / b Z, a q / c Z, a q / d Z\right)_{|x|}}, & n \text { odd } \\
\frac{1}{(a q / Z, a q / b c Z, a q / b d Z, a q / c d Z)_{|x|}}, & n \text { even }\end{cases} \\
& =\frac{(a q, a q / b e, a q / c e, a q / d e)_{N} \prod_{i=1}^{n}\left(a q / e z_{i}\right)_{N}}{Z^{N} \prod_{i=1}^{n}\left(a q z_{i} / e\right)_{N}} \\
& \times \begin{cases}\frac{e^{N}}{(a q / b Z, a q / c Z, a q / d Z, a q / e Z)_{N}}, & n \text { odd }, \\
\frac{1}{(a q / Z, a q / b e Z, a q / c e Z, a q / d e Z)_{N}}, & n \text { even } .\end{cases}
\end{aligned}
$$

Examining the proof of Theorem 4.1, we see that Corollary 4.3 is obtained by combining (3.3) with the special case $a q=b c$ of $(2.5)$, when the right-hand side is equal to $\prod_{i=1}^{n} \delta_{N_{i}, 0}$. The latter identity can be viewed as a matrix inversion [14], so Corollary 4.3 is an inverted version of Corollary 3.3, just as (2.5) is an inverted version of the standard $A$-type summation [12, Corollary 5.2].

If we let $\lambda d / a=1$ in Corollary 4.2, we obtain yet another multivariable elliptic Jackson summation. After a change of parameters, it takes the form

$$
\sum_{\substack{x_{1}, \ldots, x_{n} \geq 0 \\ x_{1}+\cdots+x_{n} \leq N}} \frac{\Delta\left(z q^{x}\right)}{\Delta(z)} \frac{\theta\left(a q^{2|x|}\right)}{\theta(a)} \frac{\prod_{1 \leq i<j \leq n}\left(z_{i} z_{j}\right)_{x_{i}+x_{j}}}{\prod_{i=1}^{n}\left(t / z_{i}\right)_{|x|-x_{i}}} \frac{\left(a, q^{-N}, b, c\right)_{|x|} \prod_{i=1}^{n}\left(t / z_{i}\right)_{|x|}}{\left(a q^{N+1}, a q / b, a q / c\right)_{|x|}} q^{|x|}
$$




$$
\begin{aligned}
& \times \prod_{i=1}^{n} \frac{\left(d z_{i}, e z_{i}, t z_{i} / d e Z^{2}\right)_{x_{i}}}{\prod_{j=1}^{n}\left(q z_{i} / z_{j}\right)_{x_{i}}} \cdot \begin{cases}\frac{1}{(a q / d Z, a q / e Z, t / Z, t / d e Z)_{|x|}}, & n \text { odd }, \\
\frac{1}{(a q / Z, a q / d e Z, t / d Z, t / e Z)_{|x|}}, & n \text { even }\end{cases} \\
& = \begin{cases}\frac{(a q, a q / b c, a q / b d Z, a q / c d Z)_{N}}{(a q / b, a q / c, a q / d Z, a q / b c d Z)_{N}}, & n \text { odd }, \\
\frac{(a q, a q / b c, a q / b Z, a q / c Z)_{N}}{(a q / b, a q / c, a q / Z, a q / b c Z)_{N}}, & n \text { even, }\end{cases}
\end{aligned}
$$

valid for $a^{2} q^{N+1}=b c d e z_{1}^{2} \cdots z_{n}^{2}$ and $t$ arbitrary. This identity is less novel than Corollary 4.3, as it can be deduced from Theorem 3.1 in a more direct manner. Indeed, writing the sum as

$$
\sum_{k=0}^{N} \sum_{\begin{array}{c}
x_{1}, \ldots, x_{n} \geq 0 \\
x_{1}+\cdots+x_{n}=k
\end{array}}(\cdots),
$$

the inner sum is computed by Theorem 3.1 and the outer sum by (2.3). In fact, the same proof gives the following more general result, which reduces to (4.2) when $(d, e)=(f Z, g Z)$ or $(Z, f g Z)$ if $n$ is odd or even, respectively.

Corollary 4.4. For parameters subject to $a^{2} q^{N+1}=b c d e, f g h z_{1}^{2} \cdots z_{n}^{2}=t$,

$$
\begin{aligned}
& \sum_{\substack{x_{1}, \ldots, x_{n} \geq 0 \\
x_{1}+\cdots+x_{n} \leq N}} \frac{\Delta\left(z q^{x}\right)}{\Delta(z)} \frac{\theta\left(a q^{2|x|}\right)}{\theta(a)} \frac{\prod_{1 \leq i<j \leq n}\left(z_{i} z_{j}\right)_{x_{i}+x_{j}}\left(a, q^{-N}, b, c, d, e\right)_{|x|}}{\left(a q^{N+1}, a q / b, a q / c, a q / d, a q / e\right)_{|x|}} q^{|x|} \\
& \quad \times \prod_{i=1}^{n} \frac{\left(t / z_{i}\right)_{|x|}\left(f z_{i}, g z_{i}, h z_{i}\right)_{x_{i}}}{\left(t / z_{i}\right)_{|x|-x_{i}} \prod_{j=1}^{n}\left(q z_{i} / z_{j}\right)_{x_{i}}} \cdot \begin{cases}\frac{1}{(f Z, g Z, h Z, t / Z)_{|x|}}, & n \text { odd }, \\
\frac{1}{(Z, f g Z, f h Z, g h Z)_{|x|}}, & n \text { even }\end{cases} \\
& =\frac{(a q, a q / b c, a q / b d, a q / c d)_{N}}{(a q / b, a q / c, a q / d, a q / b c d)_{N}} .
\end{aligned}
$$

\section{Acknowledgements}

This research is supported by the Swedish Science Research Council (Vetenskapsrådet). I would like to thank the anonymous referee for a very careful reading of the manuscript, leading to many improvements.

\section{References}

[1] Bhatnagar G., $D_{n}$ basic hypergeometric series, Ramanujan J. 3 (1999), 175-203.

[2] Bhatnagar G., Schlosser M., $C_{n}$ and $D_{n}$ very-well-poised ${ }_{10} \phi_{9}$ transformations, Constr. Approx. 14 (1998), $531-567$.

[3] Coskun H., Gustafson R.A., Well-poised Macdonald functions $W_{\lambda}$ and Jackson coefficients $\omega_{\lambda}$ on $B C_{n}$, in Jack, Hall-Littlewood and Macdonald Polynomials, Contemp. Math., Vol. 417, Amer. Math. Soc., Providence, RI, 2006, 127-155, math.CO/0412153.

[4] Date E., Jimbo M., Kuniba A., Miwa T., Okado M., Exactly solvable SOS models. II. Proof of the startriangle relation and combinatorial identities, in Conformal Field Theory and Solvable Lattice Models (Kyoto, 1986), Adv. Stud. Pure Math., Vol. 16, Academic Press, Boston, MA, 1988, 17-122. 
[5] Frenkel I.B., Turaev V.G., Elliptic solutions of the Yang-Baxter equation and modular hypergeometric functions, in The Arnold-Gelfand Mathematical Seminars, Birkhäuser Boston, Boston, MA, 1997, 171-204.

[6] Gustafson R.A., Some $q$-beta and Mellin-Barnes integrals with many parameters associated to the classical groups, SIAM J. Math. Anal. 23 (1992), 525-551.

[7] Gustafson R.A., Rakha M.A., q-beta integrals and multivariate basic hypergeometric series associated to root systems of type $A_{m}$, Ann. Comb. 4 (2000), 347-373.

[8] Milne S.C., Newcomb J.W., U(n) very-well-poised ${ }_{10} \phi_{9}$ transformations, J. Comput. Appl. Math. 68 (1996), 239-285.

[9] Rains E.M., $B C_{n}$-symmetric Abelian functions, Duke Math. J. 135 (2006), 99-180, math.CO/0402113.

[10] Rains E.M., Transformations of elliptic hypergeometric integrals, Ann. of Math. 171 (2010), 169-243, math.QA/0309252.

[11] Rosengren H., A proof of a multivariable elliptic summation formula conjectured by Warnaar, in $q$-Series with Applications to Combinatorics, Number Theory, and Physics (Urbana, IL, 2000), Contemp. Math., Vol. 291, Amer. Math. Soc., Providence, RI, 2001, 193-202, math.CA/0101073.

[12] Rosengren H., Elliptic hypergeometric series on root systems, Adv. Math. 181 (2004), 417-447, math.CA/0207046.

[13] Rosengren H., Schlosser M., Summations and transformations for multiple basic and elliptic hypergeometric series by determinant evaluations, Indag. Math. 14 (2003), 483-513, math.CA/0304249.

[14] Rosengren H., Schlosser M., Multidimensional matrix inversions and elliptic hypergeometric series on root systems, in preparation.

[15] Schlosser M., A new multivariable ${ }_{6} \psi_{6}$ summation formula, Ramanujan J. 17 (2008), 305-319, math.CA/0607122.

[16] Spiridonov V.P., Theta hypergeometric integrals, St. Petersburg Math. J. 15 (2003), 929-967, math.CA/0303205.

[17] Spiridonov V.P., Vartanov G.S., Elliptic hypergeometry of supersymmetric dualities, Comm. Math. Phys. 304 (2011), 797-874, arXiv:0910.5944.

[18] Spiridonov V.P., Warnaar S.O., New multiple ${ }_{6} \psi_{6}$ summation formulas and related conjectures, Ramanujan J. 25 (2011), 319-342.

[19] van Diejen J.F., Spiridonov V.P., Elliptic Selberg integrals, Int. Math. Res. Not. 2001 (2001), $1083-1110$.

[20] Warnaar S.O., Summation and transformation formulas for elliptic hypergeometric series, Constr. Approx. 18 (2002), 479-502, math.QA/0001006.

[21] Whittaker E.T., Watson G.N., A course of modern analysis, 4th ed., Cambridge Mathematical Library, Cambridge University Press, Cambridge, 1996. 